\title{
Follow-up infarct volume as a mediator of endovascular treatment effect on functional outcome in ischaemic stroke
}

\author{
K. C. J. Compagne ${ }^{1,2}$ (1) - A. M. M. Boers ${ }^{3,4,5} \cdot$ H. A. Marquering ${ }^{3,4} \cdot$ O. A. Berkhemer ${ }^{1,2,3} \cdot$ A. J. Yoo ${ }^{6} \cdot$ L. F. M. Beenen ${ }^{3} \cdot$ \\ R. J. van Oostenbrugge ${ }^{7,8}$ - W.H. van Zwam ${ }^{8,9}$ - Y. B. W. E. M. Roos ${ }^{10}$. C. B. Majoie ${ }^{3}$ - A. C. G. M. van Es ${ }^{1}$. \\ A. van der Lugt $^{1} \cdot$ D. W. J. Dippel ${ }^{2} \cdot$ H. Lingsma ${ }^{11} \cdot$ for the MR CLEAN Investigators
}

Received: 16 March 2018 / Revised: 21 May 2018 / Accepted: 29 May 2018 / Published online: 9 July 2018

(C) The Author(s) 2018

\begin{abstract}
Objective The putative mechanism for the favourable effect of endovascular treatment (EVT) on functional outcome after acute ischaemic stroke is preventing follow-up infarct volume (FIV) progression. We aimed to assess to what extent difference in FIV explains the effect of EVT on functional outcome in a randomised trial of EVT versus no EVT (MR CLEAN).

Methods FIV was assessed on non-contrast CT scan 5-7 days after stroke. Functional outcome was the score on the modified Rankin Scale at 3 months. We tested the causal pathway from intervention, via FIV to functional outcome with a mediation model, using linear and ordinal regression, adjusted for relevant baseline covariates, including stroke severity. Explained effect was assessed by taking the ratio of the log odds ratios of treatment with and without adjustment for FIV.

Results Of the 500 patients included in MR CLEAN, 60 died and four patients underwent hemicraniectomy before FIV was assessed, leaving 436 patients for analysis. Patients in the intervention group had better functional outcomes (adjusted common odds ratio (acOR) 2.30 (95\% CI 1.62-3.26) than controls and smaller FIV (median 53 vs. $81 \mathrm{ml}$ ) (difference $28 \mathrm{ml}$; 95\% CI 13-41). Smaller FIV was associated with better outcome (acOR per $10 \mathrm{ml} 0.60$, 95\% CI 0.52-0.68). After adjustment for FIV the effect of intervention on functional outcome decreased but remained substantial (acOR 2.05, 95\% CI 1.44-2.91). This implies that preventing FIV progression explains $14 \%$ (95\% CI 0-34) of the beneficial effect of EVT on outcome.

Conclusion The effect of EVT on FIV explains only part of the treatment effect on functional outcome.
\end{abstract}

MR CLEAN Investigators are listed in the Appendix (Online

Supplementary Table 1)

Electronic supplementary material The online version of this article (https://doi.org/10.1007/s00330-018-5578-9) contains supplementary material, which is available to authorized users.

K. C. J. Compagne

c.compagne@erasmusmc.nl

1 Department of Radiology and Nuclear Medicine, Erasmus MC University Medical Center, PO Box 2040 3000CA

Rotterdam, The Netherlands

2 Department of Neurology, Erasmus MC University Medical Center, Rotterdam, The Netherlands

3 Department of Radiology and Nuclear Medicine, Academic Medical Center (AMC), Amsterdam, The Netherlands

4 Biomedical Engineering and Physics, Academic Medical Center (AMC), Amsterdam, The Netherlands

5 Department of Robotics and Mechatronics, University of Twente, Enschede, The Netherlands
6 Division of Neurointervention, Texas Stroke Institute, Dallas-Fort Worth, TX, USA

7 Department of Neurology, Maastricht University Medical Center, Maastricht, The Netherlands

8 Cardiovascular Research Institute Maastricht, Maastricht, The Netherlands

9 Radiology, Maastricht University Medical Center, Maastricht, The Netherlands

10 Neurology, Academic Medical Center (AMC), Amsterdam, The Netherlands

11 Department of Public Health, Erasmus MC University Medical Center, Rotterdam, The Netherlands 
Key Points

- Endovascular treatment in acute ischaemic stroke patients prevents progression offollow-up infarct volume on non-contrast CT at 5-7 days.

- Follow-up infarct volume was related to functional outcome, but only explained a modest part of the effect of intervention on functional outcome.

- A large proportion of treatment effect on functional outcome remains unexplained, suggesting FIV alone cannot be used as an early surrogate imaging marker of functional outcome.

Keywords Stroke $\cdot$ Thrombectomy $\cdot$ Causality $\cdot$ Outcome $\cdot$ Biomarkers

$\begin{array}{ll}\text { Abbreviations } & \\ \text { CT } & \text { Computed tomography } \\ \text { CTA } & \text { Computed tomography angiography } \\ \text { DSA } & \text { Digital subtraction angiography } \\ \text { EVT } & \text { Endovascular treatment } \\ \text { FIV } & \text { Follow-up infarct volume } \\ \text { MR CLEAN } & \text { Multicenter Randomized } \\ & \text { Clinical Trial of Endovascular } \\ & \text { Treatment for Acute Ischemic Stroke } \\ \text { MRA } & \text { in The Netherlands } \\ \text { MRI } & \text { Magnetic resonance angiography } \\ \text { mRS } & \text { Magnetic resonance imaging } \\ \text { NCCT } & \text { Modified Rankin Scale } \\ \text { NIHSS } & \text { Non-contrast computed tomography } \\ & \text { National Institutes of Health Stroke Scale }\end{array}$

\section{Introduction}

In 2015, endovascular treatment (EVT) was shown to be effective in improving functional outcome in patients with ischaemic stroke due to intracranial large vessel occlusion [1]. Secondary outcome analyses of the randomised clinical trials also indicated significantly smaller infarct volumes at followup imaging in patients who were allocated to the intervention group [2, 3]. Studies have suggested that follow-up infarct volume (FIV) could be a useful early outcome measure [4-6].

FIV as a surrogate outcome is a well-quantifiable measure and therefore less sensitive to interobserver variability compared to clinical assessment of functional outcome such as the modified Rankin Scale score at 90 days [7, 8]. Also, FIV measurements can be assessed relatively easily and semiautomatically after treatment on non-contrast computed tomography (NCCT) or magnetic resonance imaging (MRI) scans $[9,10]$. A moderate correlation between FIV and clinical outcome has been demonstrated [11]. FIV has been suggested as a primary endpoint in late phase II clinical trials, which are intended to demonstrate an indication of therapeutic effect in promising novel treatments. Assessment of functional outcome as a clinical endpoint requires prolonged follow-up. An early surrogate marker could therefore be more feasible in clinical trials, and limit loss to follow-up $[12,13]$.
A recent post hoc study demonstrated that the beneficial effect of EVT on functional outcome could be explained by preventing progression of FIV, suggesting that the effect of intervention on functional outcome is mediated by FIV [6]. Formal testing of such a mechanism requires a causal mediation model to estimate the extent to which the treatment effect is explained by a mediator [14]. This is usually expressed as a proportion of the original treatment effect. In the context of testing a mediator as a surrogate marker, the Prentice criteria have been proposed to formally test for a causal relation between surrogate and clinical endpoints [15]. This analytical approach for estimating the causal effect of FIV on functional outcome has not yet been fully reported for EVT in acute ischaemic stroke and the extent to which the beneficial effect of intervention on functional outcome can be explained by difference in FIV is not yet known [16]. Understanding the causal pathway of this relation may provide further insight and may help in developing surrogate markers of functional outcome after EVT, and shed further light on outcome predictors that can be used for future stroke trials. The aim of this study was to assess whether and to what extent FIV on NCCT at 5-7 days' follow-up is a mediator of the effect of intervention on functional outcome in acute ischaemic stroke patients.

\section{Material and methods}

\section{Patients}

In this post-hoc analysis, we used data from the Multicenter Randomised Clinical Trial of Endovascular Treatment for Acute Ischemic Stroke in The Netherlands (MR CLEAN), which was performed at 16 Dutch stroke centres [2]. This randomised trial investigated the effect of EVT plus usual care (intervention) versus usual care only (control). In both treatment groups, administration of intravenous alteplase was allowed before randomisation. Patients had a minimal score of 2 on the National Institutes of Health Stroke Scale (NIHSS) at baseline and a radiologically confirmed proximal intracranial arterial occlusion of the anterior circulation. Follow-up imaging by computed tomography (CTA) or magnetic resonance angiography (MRA) was done at $24 \mathrm{~h}$ to assess endovascular recanalisation. 
After 5-7 days, a (NCCT) scan was acquired to assess FIV and haemorrhagic transformation. Institutional review board approval and written informed consent from all patients were obtained [2]. In the present study, patients were excluded if they died before the follow-up NCCT scans at 5-7 days or in case no NCCT was acquired before hemicraniectomy.

\section{Measures}

FIVs at 5-7 days' follow-up were semi-automatically segmented with the use of validated in-house- developed software based on intensity region growing algorithm [17]. Placement of seed points for initiating region growing in infarcted areas was done by an experienced radiologist to overcome selection of older infarctions. Segmentations were inspected and if necessary manually adjusted by two observers, who were blind to treatment allocation, as previously described [9]. FIV was calculated by multiplying the number of voxels with the voxel size. The semi-automated segmentations were highly correlated (Pearson's correlation coefficient of 0.98 ) to reference manual measurements [17]. Two examples of the semi-automatic segmentation process are shown in Fig. 2. Post-treatment functional outcome was measured on the modified Rankin Scale (mRS) at 90 days and was assessed in a standardised telephone interview by a single investigator and validated by blinded assessors. The mRS is a 7-point scale ranging from 0 (no symptoms) to 6 (death).

\section{Statistical analysis}

The confidence interval of the difference of measured median FIVs between both treatment groups was tested by bootstrapping with 1,000 replications. Statistical testing of a mechanism or pathway requires a mediation model [14]. Rather than a direct causal relationship between the independent variable (intervention or control group) and the dependent variable (functional outcome), a mediation model proposes that the independent variable influences the mediator variable (FIV on NCCT at 5-7 days), which in turn influences the dependent variable (Fig. 1).

Three requirements must be met to prove a true mediation relationship [14]:
1) The independent variable must be a significant predictor of the dependent variable (Fig. 1, pathway C).

2) The independent variable is a significant predictor of the mediator (Fig. 1, pathway A).

3) The mediator is a significant predictor of the dependent variable, while controlling for the independent variable. In other words: when treatment allocation and FIV are combined in one model to predict functional outcome (i.e. pathway A-B), FIV should still be a significant predictor, while the effect of intervention should be strongly reduced (compared to the unadjusted effect). This step is needed to prove that the effect goes (partly) through pathway A-B instead of C (Fig. 1).

According to the Prentice criteria, FIV must completely account for the net effect of intervention to be a perfect surrogate, meaning that in step 3 the effect of intervention on functional outcome should be reduced to a non-significant odds ratio [15].

In patients with missing FIVs, values of infarcted volumes were imputed based on relevant baseline covariates, allocated treatment and functional outcome [18]. Due to a skewed distribution of FIV measurements, the confidence interval of the difference of measured median FIVs between both treatment groups was constructed by bootstrapping with 1,000 replications. For the same reason, FIV was transformed to $\sqrt[3]{F I V}$ to achieve linearity for linear regression. Pathway A was tested with linear regression. Pathways B, C and A-B were tested with proportional odds regression without and with adjustments for age, sex, previous diabetes mellitus, previous ischaemic stroke, atrial fibrillation, NIHSS at baseline, occluded internal carotid artery terminus (ICA-T) occlusion, collateral status at baseline CTA, treatment with intravenous alteplase and time from stroke onset to randomisation. Effect estimates were presented as common odds ratios and betas with corresponding 95\% confidence intervals (CIs). To assess the proportion of the effect of intervention on functional outcome that was mediated by FIV, the log odds ratio of the indirect effect of intervention in pathway A-B was divided by the log odds ratio of the direct effect of intervention in pathway $C[19,20]$. The CIs for the proportion of the effect mediated were constructed with bootstrapping with 1,000 replications. In this approach, the $95 \%$ CI can exceed $0 \%$ and $100 \%$ but we
Fig. 1 Causal diagram showing the mediation model. Arrows are the causal direction or possible association

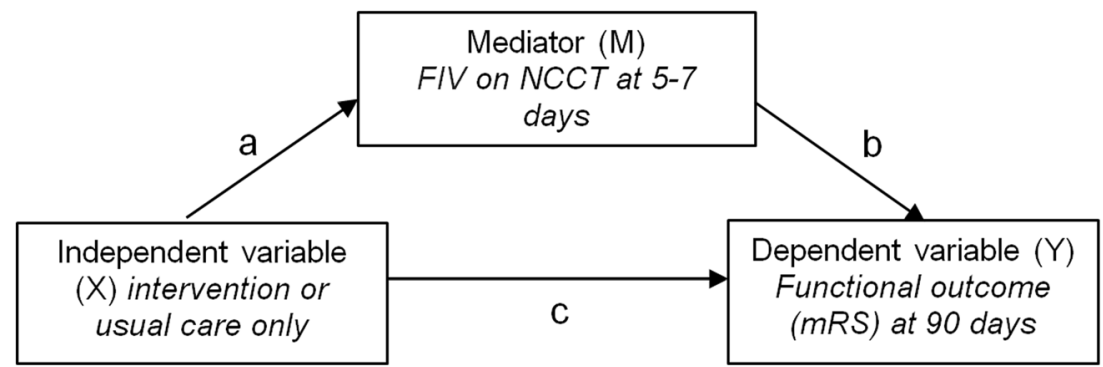


Fig. 2 Case examples of followup infarct (FIV) segmentation on non-contrast $\mathrm{CT}$, acquired between 5 and 7 days after onset. (a and b) A 56-year-old male with right-sided M1 occlusion. FIV was $292 \mathrm{ml}$ and this patient was severely disabled at 90 days (mRS 5). (c and d) A 45-yearold female with right-sided M1 occlusion. FIV was $10 \mathrm{ml}$ and the patient showed no significant disability at 90 days, despite some symptoms (mRS 1)
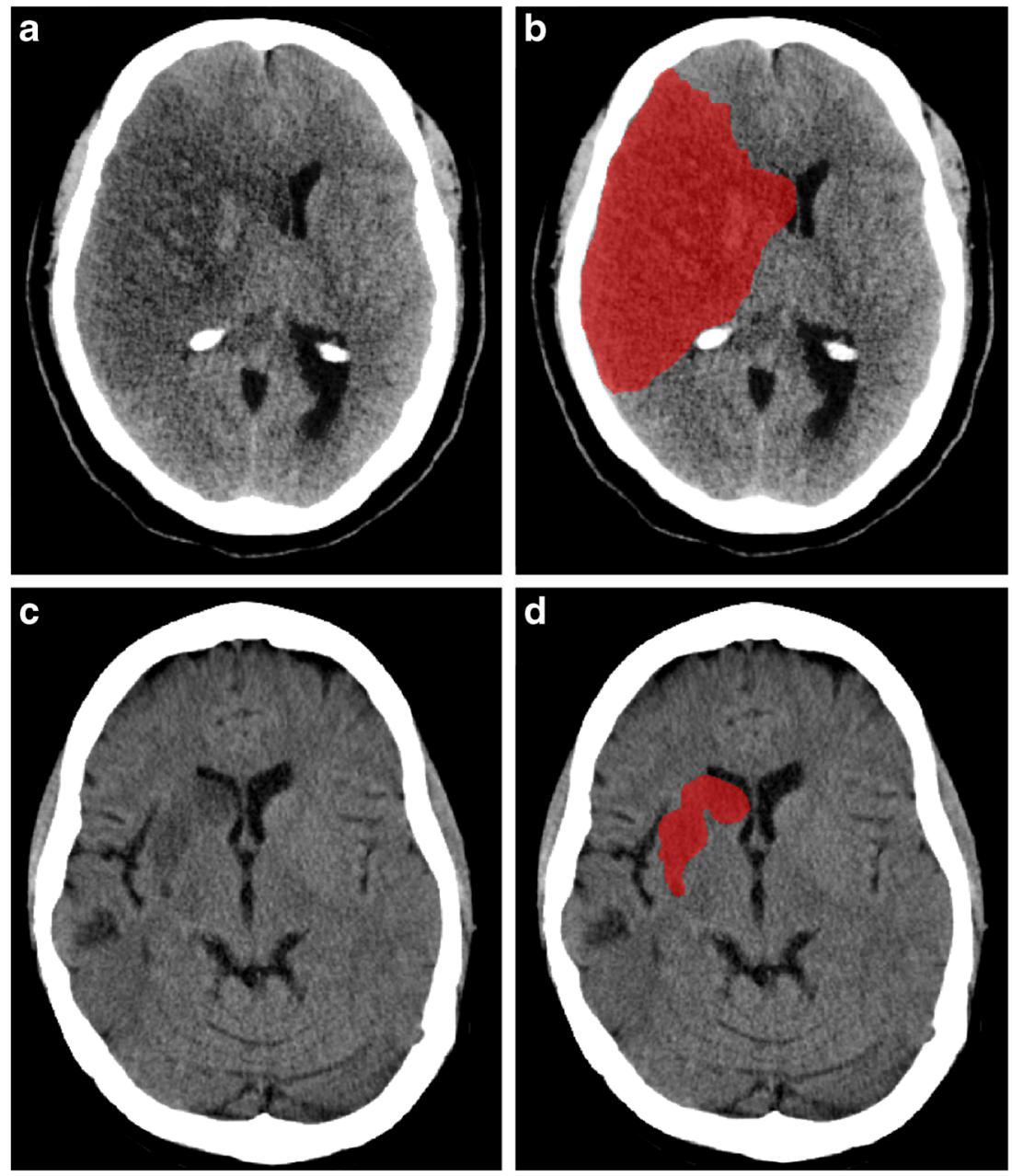

manually truncated the lower bound to $0 \%$ and the upper bound to $100 \%$.

All analyses were performed in R statistical software (version 3.4.2) with the packages foreign, rms, gvlma and boot.

\section{Sensitivity analysis}

To test the robustness of our findings against the assumptions that were made, we performed two sensitivity analyses. First, in order to account for patients who died within a week and therefore did not have a NCCT at 5-7 days and for patients who had no NCCT before hemicraniectomy, we imputed FIV in these patients with single imputation. Second, we assessed the effect of replacing missing FIV with FIV assessed from NCCT scans acquired at $24 \mathrm{~h}$.

\section{Results}

\section{Descriptives}

In total, 500 patients were included in the MR CLEAN trial. Sixty patients died before the NCCT scan at 5-7 days after initial treatment could be performed and in four patients no NCCT was performed before hemicraniectomy, leaving 436 patients for analysis (Fig. 3). In 99 (23\%) of these 436 patients no NCCT scan was made within 1 week because of logistic reasons $(n=91)$ such as transfer back to referring primary stroke centre or hospital discharge or no FIV measurement could be done because of poor scan quality $(n=8)$. Baseline characteristics of analysed patients in both treatment groups are shown in Table 1, median measured FIV in all patients was $67 \mathrm{ml}$ (IQR 30-124). Imaging outcomes regarding reperfusion on digital subtraction angiography (DSA) and recanalisation on follow-up CTA $(24 \mathrm{~h})$ of analysed patients are presented in Tables 2 and 3.

\section{Mediation analysis}

In step 1 of the mediation analysis, we tested the relationship between intervention and functional outcome. Treatment was indeed a significant predictor of functional outcome. In the present dataset, the adjusted common odds ratio (acOR) was 2.30 (95\% CI 1.62-3.26). In step 2, we tested the relationship between allocated treatment and FIV. The median FIV was 53 


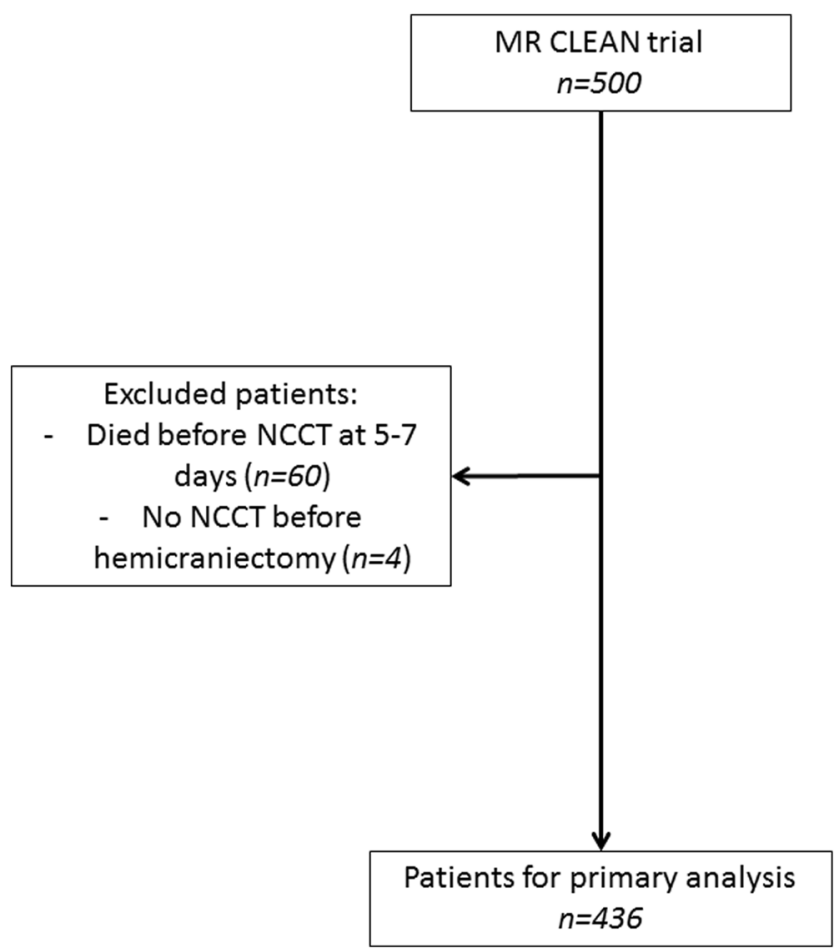

Fig. 3 Flowchart of included patients in the primary analysis

$\mathrm{ml}$ (IQR 24-116) in the intervention group and $81 \mathrm{ml}$ (IQR $35-127$ ) in the control group (difference $28 \mathrm{ml}$; 95\% CI 13-
41). Intervention was significantly related to reduction in transformed FIV with a beta of -0.37 (95\% CI $-0.65-$ -0.09). In step 3, we tested the relationship between FIV and functional outcome, with adjustment for treatment allocation. The mediator FIV was an independent variable and a significant predictor of functional outcome with cOR of $0.60(95 \%$ CI $0.52-0.67)$ per $10 \mathrm{ml}$. The direct effect of intervention on functional outcome remained statistically significant after adjustment for FIV with an acOR 2.05 (95\% CI 1.44-2.91) (Table 4). We found that preventing progression of FIV explains $14 \%$ (95\% CI 0-34) of the beneficial effect of intervention on functional outcome. All unadjusted estimates were comparable to adjusted estimates.

\section{Sensitivity analysis}

In the first sensitivity analysis, including all patients, 35 patients in the intervention group and 29 patients in the control group who died within 1 week or underwent hemicraniectomy before NCCT were additionally included by single imputation. The results of the steps were consistent with the primary analysis (Table 4). The proportion of explained mediated effect was $12 \%$ (95\% CI 0-43). In the second sensitivity analysis, the missing 5-7 days NCCT FIV were replaced by 24-h NCCT if performed instead of imputation (leaving 30 missing

Table 1 Baseline characteristics of analysed patients $(n=436)$

\begin{tabular}{llll}
\hline & $\begin{array}{l}\text { Intervention group } \\
(\mathrm{n}=198)\end{array}$ & $\begin{array}{l}\text { Control group } \\
(\mathrm{n}=238)\end{array}$ & $\begin{array}{l}\text { Excluded patients } \\
(\mathrm{n}=64)\end{array}$ \\
\hline Sex $(\mathrm{men})(\%)$ & $117(59.1)$ & $134(56.3)$ & $41(64.1)$ \\
Age $(\mathrm{y}$, median $[\mathrm{IQR}])$ & $63.47[53.3-73.4]$ & $65.67[55.1-76.4]$ & $71.40[61.8-79.9]$ \\
Pre-stroke mRS $\leq 2(\%)$ & $191(96.5)$ & $229(96.2)$ & $59(92.2)$ \\
NIHSS at baseline (median [IQR]) & $17.00[14.0-20.8]$ & $17.00(14.0-21.8)$ & $21.00[17.0-23.0]$ \\
Treatment with intravenous alteplase (\%) & $177(89.4)$ & $216(90.8)$ & $52(81.2)$ \\
Time from onset to randomisation (median $[\mathrm{IQR}])$ & $200.00[150.0-250.0]$ & $194.00[148.8-266.8]$ & $212.00[174.0-258.3]$ \\
Smoking $(\%)$ & $59(29.8)$ & $72(30.3)$ & $12(18.8)$ \\
Diabetes $(\%)$ & $23(11.6)$ & $29(12.2)$ & $16(25.0)$ \\
Atrial fibrillation $(\%)$ & $54(27.3)$ & $65(27.1)$ & $16(25.0)$ \\
Previous stroke $(\%)$ & $23(11.6)$ & $20(8.4)$ & $11(17.2)$ \\
Location of intracranial occlusion $(\%) \#$ & & & - \\
ICA & $1(0.5)$ & $3(1.3)$ & $19(29.7)$ \\
ICA terminus & $50(25.3)$ & $65(27.3)$ & $44(68.8)$ \\
M1 & $129(65.2)$ & $146(61.6)$ & $1(1.6)$ \\
M2 & $17(8.6)$ & $21(8.9)$ & - \\
A2 & $1(0.5)$ & $2(0.8)$ & $32(50.8)$ \\
ASPECTS $\geq 8(\%) \S$ & $150(75.0)$ & $194(82.6)$ & \\
\hline
\end{tabular}

§ Alberta Stroke Program Early Computed Tomography Score (ASPECTS) ranges from 0 to 10, with higher scores indicating fewer early ischaemic changes. Data were missing for three patients in the control group

\# Location of intracranial occlusion could not be assessed in one patient in the control group due to non-performed vessel imaging

$I Q R$ interquartile range 
Table 2 Explained proportions and effect sizes in the mediation analyses of the effect of intervention on functional outcome mediated by follow-up infarct volume (FIV)

\begin{tabular}{|c|c|c|c|c|c|c|c|c|c|c|}
\hline \multirow[t]{3}{*}{ Pathway* } & \multicolumn{4}{|c|}{ Unadjusted analysis } & \multicolumn{6}{|c|}{ Adjusted analyses } \\
\hline & \multirow[t]{2}{*}{$\begin{array}{l}\text { Effect } \\
\text { parameter }\end{array}$} & \multicolumn{2}{|c|}{$\begin{array}{l}\text { Primary analysis } \\
(\mathrm{n}=436)\end{array}$} & \multirow[t]{2}{*}{ Effect parameter } & \multicolumn{2}{|c|}{$\begin{array}{l}\text { Primary analysis } \\
(n=436)\end{array}$} & \multicolumn{2}{|c|}{$\begin{array}{l}\text { Sensitivity analysis } 1 \\
(\mathrm{n}=500)\end{array}$} & \multicolumn{2}{|c|}{$\begin{array}{l}\text { Sensitivity analysis } 2 \\
(\mathrm{n}=436)\end{array}$} \\
\hline & & Value & $(95 \% \mathrm{CI})$ & & Value & $(95 \% \mathrm{CI})$ & Value & $(95 \% \mathrm{CI})$ & Value & $(95 \% \mathrm{CI})$ \\
\hline$(\mathrm{X} \rightarrow \mathrm{M})$ & Beta & -0.34 & $(-0.64--0.05)$ & Beta & -0.37 & $(-0.65--0.09)$ & -0.21 & $(-0.49-0.06)$ & -0.38 & $(-0.64--0.11)$ \\
\hline$(\mathrm{M} \rightarrow \mathrm{Y})$ & $\mathrm{cOR}$ & 0.59 & $(0.52-0.66)$ & $\mathrm{acOR}$ & 0.58 & $(0.51-0.66)$ & 0.63 & $(0.56-0.70)$ & 0.49 & $(0.42-0.56)$ \\
\hline $\mathrm{C} \quad(\mathrm{X} \rightarrow \mathrm{Y})$ & $\mathrm{cOR}$ & 2.22 & $(1.58-3.13)$ & $\mathrm{acOR}$ & 2.30 & $(1.62-3.26)$ & 1.78 & $(1.29-2.46)$ & 2.30 & $(1.62-3.26)$ \\
\hline A-B $\quad(X+M \rightarrow Y)$ & $\mathrm{cOR}$ & 2.03 & $(1.44-2.86)$ & acOR & 2.05 & $(1.44-2.91)$ & 1.66 & $(1.20-2.30)$ & 2.04 & $(1.43-2.90)$ \\
\hline$\beta_{\mathrm{FIV}} \#$ & OR & 0.60 & $(0.53-0.67)$ & OR & 0.60 & $(0.52-0.67)$ & 0.66 & $(0.58-0.74)$ & 0.49 & $(0.43-0.56)$ \\
\hline Explained proportion & $\%$ & & & & 14 & $(0-34)$ & 12 & $(0-43)$ & 15 & $(0-38)$ \\
\hline
\end{tabular}

*Each pathway is shown in Fig. 1. X, independent variable (intervention- or control group). M, mediator variable (follow-up infarct volume), Y, dependent variable (functional outcome)

\# $\beta$, coefficient of FIV (mediator) in pathway A-B must be a significant predictor of the dependent variable, while controlling for the independent variable cOR common odds ratio, acOR adjusted common odds ratio, $95 \% \mathrm{CI}, 95 \%$ confidence interval

FIVs) and resulted in an explained mediated effect of $15 \%$ (95\% CI 0-38).

\section{Discussion}

In this study, we tested with mediation analysis whether the beneficial effect of intervention for acute ischaemic stroke on functional outcome could be explained by FIV. We found that FIV on NCCT at 5-7 days was affected by treatment, and was related to functional outcome, but only explained a modest part of the effect of intervention on functional outcome at 90 days measured by the modified Rankin scale in patients with

Table 3 Imaging outcomes regarding reperfusion on DSA after intervention

\begin{tabular}{lccc}
\hline \multicolumn{3}{c}{$\begin{array}{l}\text { Intervention group } \\
(\mathrm{n}=166)\end{array}$} & Control group (NA) \\
\hline $\begin{array}{l}\text { Reperfusion grades on DSA (\%) and median follow-up infarct volumes } \\
(\mathrm{ml})[\mathrm{IQR}]^{\#}\end{array}$ & \\
0 & $18(11)$ & $107[58-246]$ & - \\
1 & $9(5)$ & $137[62-222]$ & - \\
$2 \mathrm{a}$ & $32(19)$ & $55[30-118]$ & - \\
$2 \mathrm{~b}$ & $64(39)$ & $41[21-93]$ & - \\
3 & $43(26)$ & $56[35-105]$ & - \\
\hline
\end{tabular}

\# Assessed by the modified Thrombolysis in Cerebral Infarction (mTICI): 0 - no reperfusion, 1 - antegrade flow past the initial occlusion, but limited distal branch filling with little or slow distal reperfusion, $2 \mathrm{a}-$ antegrade reperfusion of less than half of the previously ischaemic territory, $2 \mathrm{~b}$ - antegrade reperfusion of more than half of the previously ischaemic territory, 3 - complete antegrade reperfusion of the previously ischaemic territory, with absence of visualised occlusion in all distal branches. Scores were not available for $32(19 \%)$ patients in the intervention group acute ischaemic stroke. This implies that FIV on NCCT only partially explains the effect of intervention on functional outcome and should therefore not be used as an early surrogate imaging marker for clinical endpoints in trials.

A previous study found a significant association between volume of FIV on NCCT and three different functional outcome measurements at 3 months. However, a moderate correlation between infarct volume and all functional outcome measures was found [11]. The study did not report the commonly used mRS score as a functional outcome measurement. Another study, which also included ischaemic stroke patients undergoing intervention, demonstrated that FIV was an important determinant of functional outcome at 3 months [4]. However, this study used imaging (NCCT or MRI) in a broad time window between $24 \mathrm{~h}$ and 2 weeks after stroke. Our conclusion also differs from a previous study on this topic, which concluded that FIV explains the effect of intervention on functional outcome [6]. However, in that study only the first and second step of mediation analysis were performed, and not the third step. This implies that no definite conclusion on mediation could be drawn, which explains the discrepancy with our findings. No other studies that reported an association between FIV and functional outcome did not perform a full a causal mediation analysis. In the REVASCAT study a mediation analysis was carried out, with similar results, but the proportion of explained treatment effect was not estimated [16]. Our study is the first full mediation analysis to analyse the pathway from intervention to FIV to functional outcome and report the proportion of explained treatment effect mediated by FIV.

Several assumptions must be made to perform an unbiased causal mediation analyses [21]. First, there is no unmeasured confounding between treatment and outcome; this assumption 
Table 4 Imaging outcomes regarding recanalisation on CTA at 24-hours

$\begin{array}{ll}\begin{array}{l}\text { Intervention group } \\ (\mathrm{n}=173)\end{array} & \begin{array}{l}\text { Control group } \\ (\mathrm{n}=190\end{array}\end{array}$

Recanalisation status on follow up CTA (\%) and median follow-up infarct volumes $(\mathrm{mL})[\mathrm{IQR}]{ }^{\#}$

\begin{tabular}{rcclr}
0 & $16(9 \%)$ & $200[65-324]$ & $59(31 \%)$ & $93[48-141]$ \\
1 & $4(2 \%)$ & $168[104-231]$ & $18(9 \%)$ & $103[82-126]$ \\
2 & $15(9 \%)$ & $24[12-61]$ & $46(24 \%)$ & $82[33-117]$ \\
3 & $138(80 \%)$ & $47[23-86]$ & $67(35 \%)$ & $51[19-82]$ \\
\hline
\end{tabular}

\# Assessed by the modified Arterial Occlusive Lesion (mAOL) score on CTA at 24 h: 0 - no recanalisation of primary intracranial occlusion, 1 incomplete or partial recanalisation of the primary intracranial occlusion without contrast passage, 2 - incomplete or partial recanalisation of the primary intracranial occlusion with contrast passage, 3 - complete recanalisation of the primary intracranial occlusion. Values were missing for $25(13 \%)$ patients in the intervention group and $48(20 \%)$ patients in the control group

$I Q R$ interquartile range

is automatically satisfied in our study due to randomisation of treatment. Secondly, no unmeasured confounding between mediator and outcome should be present. This is true for our study as the observers were blinded with respect to clinical information during imaging analysis [9]. Third, there should be no unmeasured confounding between the treatment and mediator. This requirement is also satisfied in our study due to randomisation and the fact that FIV measurements were assessed after baseline. This is also confirmed by the consistency of the results of the adjusted and unadjusted analyses.

A limitation of our study is the exclusion of deceased patients in the first week after onset and therefore missing FIV measurements at 5- to 7-day follow-up $(n=60(12 \%))$. In our sensitivity analysis, we tried to overcome this by imputing FIVs in these deceased patients. Results of the sensitivity analysis did not change the conclusions of our paper and effect sizes are comparable. Although factors other than FIV possibly play a role in early death, it is likely that the more severely affected patients with potentially large FIV will be overrepresented among patients who died early $[4,6]$. In our study, no FIV measurements on NCCT in 99 patients could be assessed at 5- to 7-day follow-up mostly because of logistic reasons; this could result in a distortion of the results. We therefore used imputation techniques to adjust for this potential bias $[22,23]$. The estimates of the mediator (FIV measurements) must be reliable and valid. Our automated, observerchecked estimation method has been shown to be reliable [17]. Overestimation of infarct size due to oedema may occur. The randomised assessment of treatment effect will reduce this bias. In a sensitivity analysis, we showed that use of 24h NCCT FIV for missing FIV did not increase the explained proportion, probably because FIV measurement is less precise, and hypodense areas may yet increase in size.
Another limitation is our relatively small sample size. The different pathways in our mediation model (EVT-FIV, FIVfunctional outcome and EVT- functional outcome) are all frequently studied and confirmed in multiple datasets. However, the proportion of the effect of EVT on functional outcome has never been calculated before. Our relatively wide CI expressed the uncertainty in this estimate. Therefore, our findings need to be replicated in other randomised control trials performed on EVT.

Our study made use of follow-up NCCT to assess FIV, because this is the most widely available and used modality. It would be of interest to also study effect mediation by FIV measured with MRI. Care should be taken, however, that selection bias in assessment does not distort the comparison between MRI and CT.

A large proportion of the treatment effect on functional outcome remains unexplained, suggesting FIV alone cannot be used as an early proxy of functional outcome. Effects of other pathways may play a role in determining functional outcome such as infarct location. Previous studies have demonstrated that certain brain regions are more sensitive than others to hypoperfusion, which may interact with FIV regarding functional outcome, given that the relevance for functional outcome varies by regional eloquence [24-26]. In our study, patients had an occlusion of the middle cerebral artery supplying eloquent brain regions. Small lesions in eloquent regions may have a larger destructive effect on functional outcome than larger infarcts in non-eloquent regions.

Further studies should address the question whether combining FIV with a measure of eloquence can increase the predictive value for functional outcome $[26,27]$. Taking eloquence into account might improve the proportion of explained mediated effect. The best method to combine eloquence, location and infarct volume is not yet known. Infarcts do not only affect the cortical regions but also white matter tracts. Small infarcts in eloquent cortical regions or important white matter tracts might result in severe strokes. This type of analysis, which takes into account the location of infarct in mediation models, requires larger datasets. In our study, we were mainly interested in FIV as a surrogate imaging biomarker as a first step, because it has been used in several studies [28]. However, for further understanding of the pathophysiological mechanisms relating infarct volume to functional outcome, taking location into account is the obvious next step [26]. Another approach could be to combine the FIV measurement with assessments of specific stroke symptoms and stroke severity. NIHSS is currently increasingly used for assessment of initial stroke severity in clinical practice [29-31], but it might also be an interesting intermediate outcome measurement [16]. Also, in our primary analysis, we assessed FIV at 5-7 days. FIV on NCCT in other time windows might also be of interest as a surrogate marker [32]. Finally, the use of more advanced imaging modalities such 
as MRI to determine FIV would be interesting for future studies.

In conclusion, we confirmed that intervention prevents progression of FIV on NCCT, but this only partly explains the beneficial effect of intervention on functional outcome.

Funding The MR CLEAN trial was partly funded by the Dutch Heart Foundation and by unrestricted grants from AngioCare BV, Medtronic/ Covidien/EV3®, MEDAC Gmbh/LAMEPRO, Penumbra Inc., Stryker®, and Top Medical/Concentric. The MR CLEAN is registered under number NTR1 804 in the Dutch trial register and under ISRCTN10888758 in the ISRCTN register. Erasmus Medical Center Rotterdam received funds from Stryker® for consultations by A. van der Lugt, A. van Es and D. Dippel and from Bracco Imaging ${ }^{\circledR}$ for consultations by D. Dippel. Academic Medical Center Amsterdam received funds from Stryker® for consultations by C. Majoie, Y. Roos and O. Berkhemer. Maastricht University Medical Center received funds from Stryker® and Cerenovus ${ }^{\circledR}$ for consultations by W. Zwam.

\section{Compliance with ethical standards}

Guarantor The scientific guarantor of this publication is Kars C.J. Compagne.

Conflict of interest A. Boers and H. Marquering own stock in Nico-lab B.V. A. Yoo reports research grants form Penumbra Inc. and Neuravi Inc., and consultant fees from Cerenovus/J\&J. No other personal disclosures are reported.

Statistics and biometry $\mathrm{H}$. Lingsma and K. Compagne performed statistical analysis for this study. One of the authors (H. Lingsma) has significant statistical expertise.

Informed consent Written informed consent was obtained from all subjects (patients) in this study.

Ethical approval Institutional Review Board approval was obtained.

Study subjects or cohorts overlap Data were obtained from the MR CLEAN trial. Multiple studies have been performed with the data from the MR CLEAN trial. However, our data have not been reported in any other paper, sub-study or abstract.

\section{Methodology \\ - prospective \\ - randomised controlled trial \\ - multicentre study}

Open Access This article is distributed under the terms of the Creative Commons Attribution 4.0 International License (http:// creativecommons.org/licenses/by/4.0/), which permits unrestricted use, distribution, and reproduction in any medium, provided you give appropriate credit to the original author(s) and the source, provide a link to the Creative Commons license, and indicate if changes were made.

\section{References}

1. Goyal M, Menon BK, van Zwam WH et al (2016) Endovascular thrombectomy after large-vessel ischaemic stroke: a meta-analysis of individual patient data from five randomized trials. Lancet 387(10029):1723-1731
2. Berkhemer OA, Fransen PS, Beumer D et al (2015) A randomized trial of intraarterial treatment for acute ischemic stroke. N Engl $\mathrm{J}$ Med 372(1):11-20

3. Jovin TG, Chamorro A, Cobo E et al (2015) Thrombectomy within 8 hours after symptom onset in ischemic stroke. N Engl J Med 372(24):2296-2306

4. Yoo AJ, Chaudhry ZA, Nogueira RG et al (2012) Infarct volume is a pivotal biomarker after intra-arterial stroke therapy. Stroke 43(5): 1323-1330

5. Barrett KM, Ding YH, Wagner DP, Kallmes DF, Johnston KC, Investigators A (2009) Change in diffusion-weighted imaging infarct volume predicts neurologic outcome at 90 days: results of the Acute Stroke Accurate Prediction (ASAP) trial serial imaging substudy. Stroke 40(7):2422-2427

6. Al-Ajlan FS, Goyal M, Demchuk AM et al (2016) Intra-Arterial Therapy and Post-Treatment Infarct Volumes: Insights From the ESCAPE Randomized Controlled Trial. Stroke 47(3):777-781

7. Quinn TJ, Dawson J, Walters MR, Lees KR (2009) Exploring the reliability of the modified rankin scale. Stroke 40(3):762-766

8. Quinn TJ, Dawson J, Walters MR, Lees KR (2008) Variability in modified Rankin scoring across a large cohort of international observers. Stroke 39(11):2975-2979

9. Bucker A, Boers AM, Bot JCJ et al (2017) Associations of Ischemic Lesion Volume With Functional Outcome in Patients With Acute Ischemic Stroke: 24-Hour Versus 1-Week Imaging. Stroke 48(5): 1233-1240

10. Lansberg MG, Lee J, Christensen S et al (2011) RAPID automated patient selection for reperfusion therapy: a pooled analysis of the Echoplanar Imaging Thrombolytic Evaluation Trial (EPITHET) and the Diffusion and Perfusion Imaging Evaluation for Understanding Stroke Evolution (DEFUSE) Study. Stroke 42(6): $1608-1614$

11. Saver JL, Johnston KC, Homer D et al (1999) Infarct volume as a surrogate or auxiliary outcome measure in ischemic stroke clinical trials. The RANTTAS Investigators. Stroke 30(2):293-298

12. Ebinger M, Christensen S, De Silva DA et al (2009) Expediting MRI-based proof-of-concept stroke trials using an earlier imaging end point. Stroke 40(4):1353-1358

13. Barber PA, Parsons MW, Desmond PM et al (2004) The use of PWI and DWI measures in the design of "proof-of-concept" stroke trials. J Neuroimaging 14(2):123-132

14. Baron RM, Kenny DA (1986) The moderator-mediator variable distinction in social psychological research: conceptual, strategic, and statistical considerations. J Pers Soc Psychol 51(6):1173-1182

15. Prentice RL (1989) Surrogate endpoints in clinical trials: definition and operational criteria. Stat Med 8(4):431-440

16. Al-Ajlan FS, Al Sultan AS, Minhas P et al (2018) Posttreatment Infarct Volumes when Compared with 24-Hour and 90-Day Clinical Outcomes: Insights from the REVASCAT Randomized Controlled Trial. AJNR Am J Neuroradiol 39(1):107-110

17. Boers AM, Marquering HA, Jochem JJ et al (2013) Automated cerebral infarct volume measurement in follow-up noncontrast CT scans of patients with acute ischemic stroke. AJNR Am J Neuroradiol 34(8):1522-1527

18. Moons KG, Donders RA, Stijnen T, Harrell FE Jr (2006) Using the outcome for imputation of missing predictor values was preferred. $\mathrm{J}$ Clin Epidemiol 59(10):1092-1101

19. Vanderweele TJ, Vansteelandt S (2010) Odds ratios for mediation analysis for a dichotomous outcome. Am J Epidemiol 172(12): $1339-1348$

20. Freedman LS, Graubard BI, Schatzkin A (1992) Statistical validation of intermediate endpoints for chronic diseases. Stat Med 11(2): $167-178$

21. Valeri L, Vanderweele TJ (2013) Mediation analysis allowing for exposure-mediator interactions and causal interpretation: 
theoretical assumptions and implementation with SAS and SPSS macros. Psychol Methods 18(2):137-150

22. Dong Y, Peng CY (2013) Principled missing data methods for researchers. Springerplus 2(1):222

23. van der Heijden GJ, Donders AR, Stijnen T, Moons KG (2006) Imputation of missing values is superior to complete case analysis and the missing-indicator method in multivariable diagnostic research: a clinical example. J Clin Epidemiol 59(10):1102-1109

24. Payabvash S, Souza LC,Wang Y et al (2011) Regional ischemic vulnerability of the brain to hypoperfusion: the need for location specific computed tomography perfusion thresholds in acute stroke patients. Stroke 42(5):1255-1260

25. Marcoux FW, Morawetz RB, Crowell RM, DeGirolami U, Halsey JH Jr (1982) Differential regional vulnerability in transient focal cerebral ischemia. Stroke 13(3):339-346

26. Ernst M, Boers AMM, Aigner A et al (2017) Association of Computed Tomography Ischemic Lesion Location With Functional Outcome in Acute Large Vessel Occlusion Ischemic Stroke. Stroke 48(9):2426-2433

27. Rangaraju S, Streib C, Aghaebrahim A, Jadhav A, Frankel M, Jovin TG (2015) Relationship Between Lesion Topology and Clinical
Outcome in Anterior Circulation Large Vessel Occlusions. Stroke 46(7):1787-1792

28. Simonsen CZ, Yoo AJ, Sorensen LH et al (2018) Effect of general anesthesia and conscious sedation during endovascular therapy on infarct growth and clinical outcomes in acute ischemic stroke: a randomized clinical trial. JAMA Neurol 75(4):470-477

29. Brott T, Adams HP Jr, Olinger CP et al (1989) Measurements of acute cerebral infarction: a clinical examination scale. Stroke 20(7): 864-870

30. Adams HP Jr, Davis PH, Leira EC et al (1999) Baseline NIH Stroke Scale score strongly predicts outcome after stroke: A report of the Trial of Org 10172 in Acute Stroke Treatment (TOAST). Neurology 53(1):126-131

31. Frankel MR, Morgenstern LB, Kwiatkowski T et al (2000) Predicting prognosis after stroke: a placebo group analysis from the National Institute of Neurological Disorders and Stroke rt-PA Stroke Trial. Neurology 55(7):952-959

32. Lansberg MG, O'Brien MW, Tong DC, Moseley ME, Albers GW (2001) Evolution of cerebral infarct volume assessed by diffusionweighted magnetic resonance imaging. Arch Neurol 58(4):613-617 